\title{
ASPECTOS CLÍNICOS E EVOLUTIVOS DA HIDROCEFALIA NA NEUROCISTICERCOSE
}

\author{
Svetlana Agapejev', Ana Flávia P. Pouza², Rodrigo Bazan³, Antonio Tadeu S. Faleiros ${ }^{4}$
}

\begin{abstract}
RESUMO - Com o propósito de analisar os aspectos clínicos da hidrocefalia (HDC) na neurocisticercose (NCC), realizou-se o estudo retrospectivo de 47 prontuários de pacientes com HDC e NCC. Verificou-se que 70,2\% eram homens, entre 21 e 50 anos. A hipertensão intracraniana (HIC) ocorreu em todos os pacientes, cefaléia (CEF) em $89,4 \%$, meningoencefalite (ME) em $80,8 \%$ e distúrbios psíquicos (PSI) em 74,5\%. A síndrome liquórica da NCC foi detectada em $65,9 \%$ pacientes. Além da HDC, as tomografias computadorizadas de crânio (TC) mostraram lesões císticas e edema cerebral difuso em 59,6\% cada, calcificações em 55,3\%. Dos 41 pacientes $(87,2 \%)$ com derivação ventriculoperitoneal (DVP), em $22(53,7 \%)$ deles foram necessárias uma a sete revisões/paciente (média=3). A evolução foi satisfatória em $51,1 \%$ e fatal em $31,9 \%$. Conclui-se que a hidrocefalia é mais comum no sexo masculino em idade produtiva, tendo a HIC, CEF, MN e PSI como manifestações freqüentes e que, a necessidade de revisões de DVP, piora o prognóstico.
\end{abstract}

PALAVRAS-CHAVE: aspectos clínicos, hidrocefalia, neurocisticercose.

\section{Clinical and evolutive aspects of hydrocephalus in neurocysticercosis}

\begin{abstract}
With the purpose to verify clinical aspects of hydrocephalus $(\mathrm{HC})$ in patients with neurocysticercosis (NCC), a retrospective study of 47 patients was performed. The majority of patients $(70.2 \%)$ were men aging 21-50 years. Intracranial hypertension (ICH) occurred in all patients, headache (HA) in 42 (89.4\%), meningoencephalitis (ME) in $38(80.8 \%)$ and psychiatric disorders (PD) in 34 (72.3\%). The cerebrospinal fluid syndrome of NCC was detected in 31 patients (65.9\%). In addition to HC, computed tomography (CT) scans showed cystic lesions in $28(59.6 \%)$ patients, diffuse brain edema also in 28 (59.6\%), and calcifications in 26 $(55.3 \%)$. Shunts were inserted in $41(87.2 \%)$ patients and $22(53.7 \%)$ of them were submitted to 1-7 surgical revision/patient $($ mean $=3$ ) that were higher $(\operatorname{mean}=4)$ in those who died than in survivors $(\operatorname{mean}=2)$. Evolution was satisfactory in $24(51.1 \%)$ patients and fatal in $15(31.9 \%)$. It is possible to conclude that, in patients with NCC, HC occurs predominantly in men in productive life with ICH, HA, ME and PD as common manifestations, and the need for shunt revision makes patient's prognosis worse.
\end{abstract}

KEY WORDS: clinical aspects, hydrocephalus, neurocysticercosis.

A ventriculomegalia (VM) ou hidrocefalia (HDC), como é mais conhecida, caracteriza-se por aumento de volume do líquido cefalorraqueano (LCR) associado a dilatação dos ventrículos cerebrais. Dependendo do mecanismo responsável pela formação dessa dilatação, classifica-se em HDC ex-vácuo (VM conseqüente à perda de parênquima cerebral sem alteração na pressão liquórica), HDC de pressão normal (VM conseqüente à redução da vazão liquórica por defeito na absorção de LCR e/ou insuficiência do espaço subaracnóideo), HDC comunicante (VM conseqüente à secreção excessiva de LCR, insuficiência venosa ou alteração da absorção do LCR) e HDC não-comunicante ou obstrutiva (VM conseqüente à obstrução do fluxo liquórico causada pela presença de processo expansivo ou por fibrose pós-inflamatória) ${ }^{1}$.

A neurocisticercose (NCC), infecção do sistema nervoso central (SNC) pela forma larvária metacestóide da Taenia sp caracteriza-se por um polimorfismo clínico e laboratorial, o que leva a dificuldades para o seu diagnóstico. Incide em ambos os sexos, com discreto predomínio em homens entre os 21 e 40 anos, mas ocorrendo em qualquer faixa etária². A manifestação dos sintomas é resultante da interação parasita-hospedeiro e da intensidade na resposta reacional inflamatória ao redor do parasita e longe dele (rea-

Departamento de Neurologia e Psiquiatria da Faculdade de Medicina de Botucatu da Universidade Estadual Paulista Júlio de Mesquita Filho, Botucatu SP, Brasil (UNESP): ${ }^{1}$ Professora Adjunta da Disciplina de Neurologia Clínica; ${ }^{2}$ Médica; ${ }^{3}$ Professor Temporário da Disciplina de Neurologia Clínica; ${ }^{4}$ Professor Doutor da Disciplina de Neurocirurgia. A apresentação deste estudo, como pôster, durante o XI Congresso Panamericano de Neurologia, realizado em Santiago (Chile), foi patrocinada pela Fundação para o Desen-

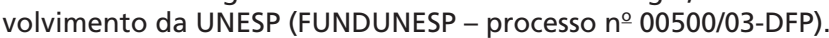

Recebido 11 Maio 2006, recebido na forma final 30 Março 2007. Aceito 30 Abril 2007.

Dra. Svetlana Agapejev - Departamento de Neurologia e Psiquiatria / Faculdade de Medicina / UNESP - 18618-000 Botucatu SP - Brasil. E-mail: agapejev@fmb.unesp.br 
ção à distância). Na NCC, as reações à distância estão associadas às situações graves e incapacitantes ${ }^{3}$. Destas, a hipertensão intracraniana (HIC), que ocorre em 36 a $66 \%$ dos pacientes, resulta da HDC, a causa mais comum em 18 a $91 \%$ das vezes ${ }^{4}$, caracterizando um grupo extremamente heterogêneo. Ou seja, a HDC por NCC resulta de uma interação entre múltiplos mecanismos resultantes do processo inflamatório pericístico, à distância do local de fixação do cisticerco e à presença de cistos intraventriculares ${ }^{4-6}$.

O propósito deste estudo foi analisar os aspectos clínicos que envolvem pacientes com HDC e portadores de NCC.

\section{MÉTODO}

Casuística - Efetuou-se levantamento das interposições de sistema de derivação ventriculoperitoeal (DVP) realizadas em pacientes internados no Hospital das Clínicas de Botucatu da Universidade Estadual Paulista (HC-UNESP) no período de setembro 1984 a agosto 1999, relacionadas no livro de cirurgias do Serviço de Neurocirurgia.

Critérios de inclusão - De um total de 138 pacientes listados, foram selecionados 47 que tinham o diagnóstico de NCC, receberam esquema terapêutico com albendazol ${ }^{5} \mathrm{e}$ não apresentavam qualquer um dos critérios de exclusão.

Critérios de exclusão - Idade inferior a 12 anos, prontuários incompletos, ausência ou impossibilidade de encontrar as respectivas tomografias computadorizadas (TC) de crânio e ressonâncias magnéticas (RM), ausência de registro de exame de LCR antes e após tratamento cisticida, presença de outras patologias associadas à NCC.

Tipo de estudo realizado - Nos 47 pacientes selecionados, com HDC e NCC, realizou-se estudo retrospectivo, através da revisão de seus prontuários, em que foram analisados: gênero, idade, manifestações clínicas, exames complementares (LCR, TC), interposição de DVP e evolução clínica.

Diagnóstico de NCC - Baseou-se nos critérios de Del Brutto et $\mathrm{al}^{6}$.

Diagnóstico de HDC - Obteve-se através de arteriografia carotídea em 4 (8,5\%) pacientes e TC crânio em 43 (91,5\%), utilizando-se o índice de Evans ${ }^{7}$.

Estatística - Por orientação do estaticista, os dados obtidos foram expressos em porcentagem.

\section{Unitermos}

Síndrome liquórica da NCC - Presença concomitante de pleocitose $>5$ células $/ \mathrm{mm}^{3}$, eosinofilorraquia $\geq 2 \%$, hiperproteinorraquia, hipoglicorraquia e positividade das reações para cisticercose ${ }^{8}$.

Edema cerebral difuso - Imagens em TC e RM mostrando diminuição ou desaparecimento de sulcos corticais e cisternas da base do crânio com diminuição volumétrica do sistema ventricular, diminuição da diferenciação entre substância branca e cinzenta, podendo haver captação difusa do contraste.

Edema transependimário - Imagens em TC e RM sugerindo presença de LCR no espaço em torno dos ventrículos, principalmente, em volta dos cornos frontais e occipitais.

Aracnoidite de IVo ventrículo - Imagens em TC e RM mostrando fibrose cística reacional com VM e deformação de IVo ventrículo.

IVo ventrículo isolado - Imagens em TC e RM mostrando VM de IVo ventrículo associada a normalidade dos demais ventrículos.

Aracnoidite de base - Imagens em RM mostrando fibrose nas cisternas da base do crânio.

Aracnoidite medular - Imagens em RM mostrando fibrose cística no canal medular.

Crise epiléptica parcial simples - Manifestações motoras, somato-sensoriais, autonômicas e psíquicas, sem alteração de consciência; crise epiléptica parcial complexa: manifestações de automatismos e de atividade motora complexa não dirigida e inadequada, com alteração da consciência e, no período pós-ictal, confusão mental e desorientação; crise epiléptica com generalização secundária: convulsões tônico-clônicas generalizadas, com perda de consciência, precedidas por crises parciais simples ou complexas; crise epiléptica generalizada primária: convulsões tônico-clônicas clinicamente generalizadas desde o início e, no período pós-ictal, confusão mental e desorientação ${ }^{9}$.

Índice de Evans - Relação entre a medida das distâncias dos pólos frontais dos ventrículos laterais e a medida da largura interna do crânio. Relação maior que 0,30 determinou o diagnóstico de $\mathrm{HDC}^{7}$.

\section{RESULTADOS}

Freqüência de HDC por NCC - No Serviço de Neurologia/Neurocirurgia do HC/UNESP de Botucatu, verificou-se freqüência de 0,3\% em crianças até 12 anos e de $34,1 \%$ em indivíduos acima dessa faixa etária.

Diagnóstico de NCC - O diagnóstico de NCC foi considerado definitivo em $33(70,2 \%)$ pacientes, provável em $10(21,3 \%)$ e, possível, em 4 (8,5\%).

Gênero - $33(70,2 \%)$ pacientes eram homens e 14 $(29,8 \%)$, mulheres.

Idade - A faixa etária variou de 14 a 62 anos, predominando $(91,5 \%)$ entre 21 e 60 anos, principalmente, dos 21 aos 40 anos $(57,4 \%)$.

Manifestações clínicas - Todos os quadros clínicos (Tabela 1) estiveram associados entre si. Na primeira consulta, todos os pacientes apresentavam HIC de gravidade variável. Dos distúrbios psiquiátricos, os sinais e sintomas de depressão (apatia, isolamento, sonolência aumentada, diminuição de ingestão alimen- 
Tabela 1. Manifestações clínicas de 47 pacientes com hidrocefalia e neurocisticercose.

\begin{tabular}{lcc}
\hline Manifestação clínica & $\mathrm{N}^{\circ}$ de pacientes & $\%$ \\
\hline Hipertensão intracraniana & 47 & 100 \\
Cefaléia $^{\mathrm{a}}$ & 42 & 89,4 \\
Meningoencefalite/meningite $^{\mathrm{b}}$ & 38 & 80,8 \\
Distúrbios psíquicos $^{\mathrm{b}}$ & 34 & 72,3 \\
Epilepsiac $^{c}$ & 23 & 48,9 \\
Acidente vascular cerebral $^{\text {Compressão medular }}{ }^{\mathrm{C}}$ & 8 & 17,0 \\
Compressão radicular & 4 & 8,5 \\
\hline
\end{tabular}

${ }^{\mathrm{a}}$ Tipo tensional e tipo migrânea; ${ }^{\mathrm{b}} \mathrm{A}$ depressão manifestou-se em $24(51,1 \%)$ pacientes, como primeiro sintoma ou de aparecimento durante a evolução clínica. A síndrome demencial ocorreu em $4(8,5 \%)$ pacientes, durante a evolução clínica; 'As crises parciais com generalização secundária ocorreram em $19(82,6 \%)$ pacientes, as crises generalizadas primárias em dois $(8,7 \%)$ e crises parciais simples em outros dois $(8,7 \%)$. Quatro $(8,5 \%)$ pacientes apresentaram mais de um tipo de crise epiléptica; ${ }^{\mathrm{d} A r a c n o i d i t e ~ c i ́ s t i c a ~ e m ~ t r e ̂ s ~ p a c i e n t e s ~ e ~ c i s t o ~ e x t r a m e d u l a r ~ t o r a ́ c i c o ~ e m ~ u m . ~}$

Tabela 2. LCR nos 47 pacientes com hidrocefalia e neurocisticercose.

\begin{tabular}{lcc}
\hline Parâmetro do LCR & $\mathrm{N}^{\circ}$ de pacientes & $\%$ \\
\hline Proteínas >40 mg/dL & 44 & 93,6 \\
Celularidade >5 células/mm ${ }^{3}$ & 41 & 87,2 \\
Neutrófilos & 35 & 74,5 \\
Plasmócitos >1\% & 28 & 59,6 \\
Eosinófilos > $\%$ & 27 & 57,4 \\
Reações positivas para cisticercose* & 32 & 68,1 \\
Glicose <40 mg/dL & 30 & 63,8 \\
\hline
\end{tabular}

*Reação de fixação de complemento, imunofluorescência e/ou ELISA.

Obs: A síndrome liquórica da NCC foi detectada em 31 pacientes (65,9\%).

Tabela 3. Alterações em tomografia computadorizada de crânio (TC) em 47 pacientes com hidrocefalia e neurocisticercose.

\begin{tabular}{lcc}
\hline Alterações & No $^{\circ}$ de pacientes & $\%$ \\
\hline Hidrocefalia & 47 & 100 \\
Edema cerebral difuso $^{\text {a }}$ & $28^{\text {a }}$ & 59,6 \\
Cisto (s) $^{\text {b }}$ & $28^{\text {a }}$ & 59,6 \\
Calcificação(ões) & 13 & 27,6 \\
Associação cisto(s) e calcificação(ões) & 15 & 31,9 \\
Acidente vascular cerebral & 8 & 17,0 \\
\hline
\end{tabular}

${ }^{a}$ Dois $(4,2 \%)$ pacientes apresentaram HDC e edema cerebral difuso, sem a presença de lesões sugestivas de cisticercose, sendo o diagnóstico de NCC obtido através das reações imunológicas no LCR; 'DObservaram-se cistos sem realce pelo contraste em $11(23,4 \%)$, com realce em $7(14,9 \%)$ e cistos racemosos (CR) em $10(21,3 \%)$. A presença exclusiva de CR ocorreu em $2(4,2 \%)$ pacientes e, sua associação a calcificações e/ou vesícula(s), em 8 (17,0\%).

tar/anorexia, choro fácil) foram os mais freqüentes, como primeiro sintoma ou de aparecimento durante a evolução clínica. As manifestações de demência surgiram apenas durante a evolução clínica.

\section{Exames complementares}

$L C R$ - Na Tabela 2 está expressa a distribuição dos pacientes conforme as alterações apresentadas no
LCR. Com exceção dos LCRs de pacientes em vigência de ventriculite purulenta pós-DVP, a celularidade variou de $0-680$ células $/ \mathrm{mm}^{3}$ às custas de $0-45 \%$ neutrófilos, 0-65\% eosinófilos, 0-99\% linfócitos, 0-33\% monócitos e 0-30\% plasmócitos. Em 20 (42,5\%) pacientes, a eosinofilorraquia não ocorreu ou foi $\leq 2 \%$. A glicorraquia oscilou de 0-124 mg/dL e, em um ou mais LCRs de $8(17,0 \%)$ pacientes, não foi detectável 


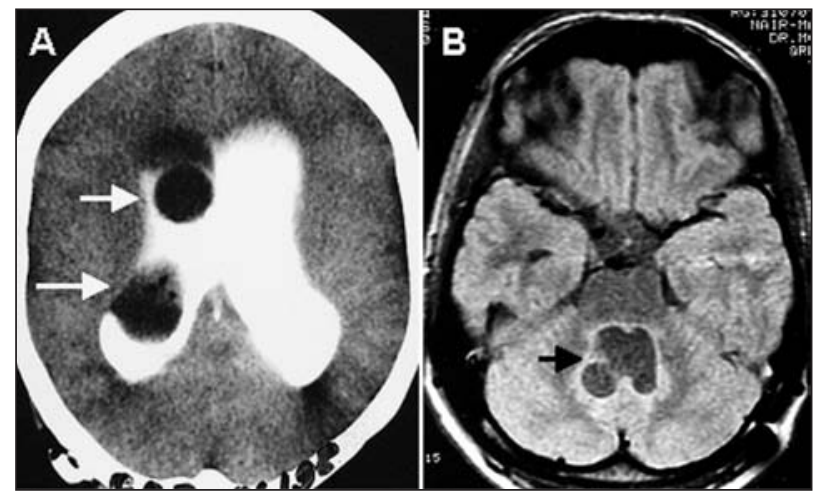

Fig 1. (A) Mulher, 23 a, hipertensão intracraniana e meningoencefalites de repetição, síndrome $L C R$ na NCC, DVP com várias revisões realizadas fora do HC-UNESP, evolução satisfatória: tomoventriculografia mostrando acentuada hidrocefalia associada a edema cerebral e presença de cistos em ventrículo lateral direito (setas). (B) Homem, 40 a, meningoencefalite + hipertensão intracraniana + cefaléia tipo tensional, síndrome $L C R$ na NCC, DVP sem revisões, evolução satisfatória: $R M$ mostra cisticerco racemoso (seta) em IV ventrículo identificado após DVP.

(glicorraquia=zero). A proteinorraquia variou de 8,58289 mg/dL. Em 15 (31,9\%) pacientes as reações imunológicas para cisticercose, no LCR, não foram realizadas ou mostraram-se não reagentes. Excluindo esses 15 pacientes, todos os demais apresentaram positividade das reações para cisticercose que ocorreu na proporção média de um LCR positivo para cada duas amostras de LCR colhidas/paciente.

Imagem - Na Tabela 3 está expressa a distribuição dos pacientes conforme as lesões encontradas nos exames de TC de crânio. A HDC, subjetivamente, foi considerada discreta em $3(6,4 \%)$ pacientes e moderada/acentuada em $44(93,6 \%)$. A assimetria ventricular foi vista em $4(8,5 \%)$ pacientes, dois deles com discreta HDC e, os outros dois, com acentuada. A tomoventriculografia (Fig 1A) permitiu identificar cistos intraventriculares, não detectados à RM, em dois pacientes.

A RM, realizada em 21 (44,7\%) pacientes, confirmou e definiu com mais precisão os achados à TC em $10(47,6 \%)$. Nos outros $11(52,4 \%)$, foram identificados, através da RM, cistos intraventriculares (Fig 1B) em dois deles, infartos múltiplos em um, cistos parenquimatosos em um, cistos em cisterna magna em um e aracnoidite de base do crânio (Fig 2A) em um e, através da RM de medula, presença de cisto medular torácico associado à aracnoidite em uma paciente e, aracnoidite cística medular (Fig 2B), em outros três.

Interposição de DVP - Foi realizada em 41 (87,2\%) pacientes, utilizando-se sistemas de média pressão de

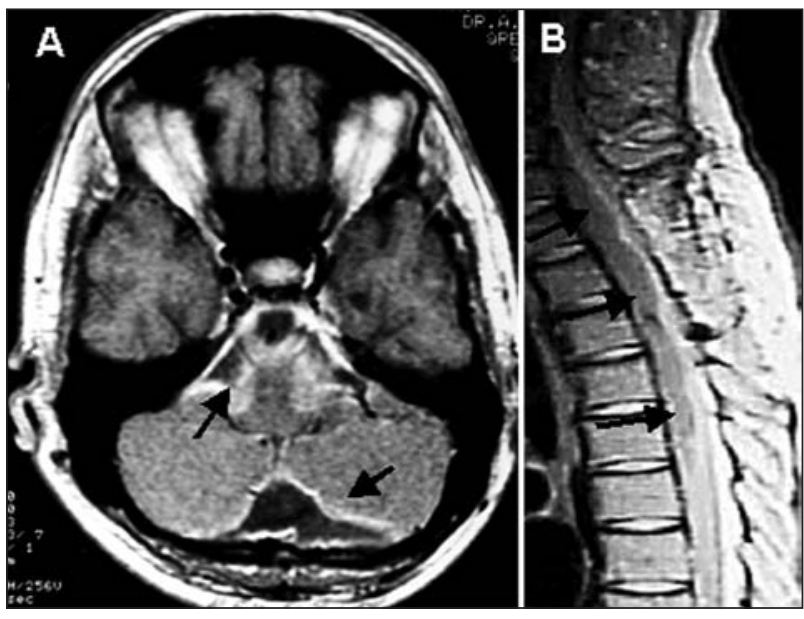

Fig 2. (A) Homem, 25 a, meningoencefalite + hipertensão intracraniana + cefaléia tipo migrânea, síndrome LCR na NCC, DVP com seis revisões, óbito: $R M$ mostrando (setas) intensa aracnoidite de base do crânio. (B) mulher, 24 a, meningoencefalite + hipertensão intracraniana + paraplegia espástica + HDC antes do comprometimento medular, LCR apenas com hiperproteinorraquia, DVP com uma revisão, óbito: RM mostrando (setas) intensa aracnoidite medular comprovada durante ato cirúrgico.

marcas variáveis. As DVPs foram instaladas antes do tratamento cisticida em $31(65,9 \%)$ pacientes, durante em 2 (4,2\%) e, após, em 8 (17,0\%). Em 22 (53,6\%) pacientes com DVP, houve necessidade de revisões do sistema por $1-7$ vezes (média=3 revisões/paciente). 0 número de revisões foi maior (média=3,8 revisões/paciente) nos pacientes que faleceram e menor nos sobreviventes (média $=2,3$ revisões/paciente).

Evolução clínica - Foi satisfatória em 24 (51,1\%) pacientes, fatal em 15 (31,9\%) e seqüelar em $8(17,0 \%)$. Na paciente com cisticercose medular, o diagnóstico de HDC foi obtido 19 meses após a definição do comprometimento medular. Nos pacientes com aracnoidite cística medular, este diagnóstico foi obtido entre 8 a 11 meses após a DVP.

Os óbitos ocorreram num período de 3 dias a 12 meses após a DVP. Sete $(16,1 \%)$ pacientes apresentaram ventriculite purulenta pós-DVP. Todos os seis pacientes sem DVP apresentaram evolução satisfatória, independente do grau de HDC. Seis pacientes $(14,6 \%)$ desenvolveram aumento do IVo ventrículo isolado. Destes, quatro $(66,7 \%)$ faleceram e, nos outros dois $(33,3 \%)$ que sobreviveram, verificou-se regressão do tamanho do IVo ventrículo. Desde a alta hospitalar e durante a evolução ambulatorial, nove $(28,1 \%)$ pacientes permaneceram assintomáticos e sem medicação.

Após o esquema cisticida, num período superior a um mês e inferior a 24 meses, oito $(17,0 \%)$ pacientes foram submetidos a neurocirurgia: um para des- 

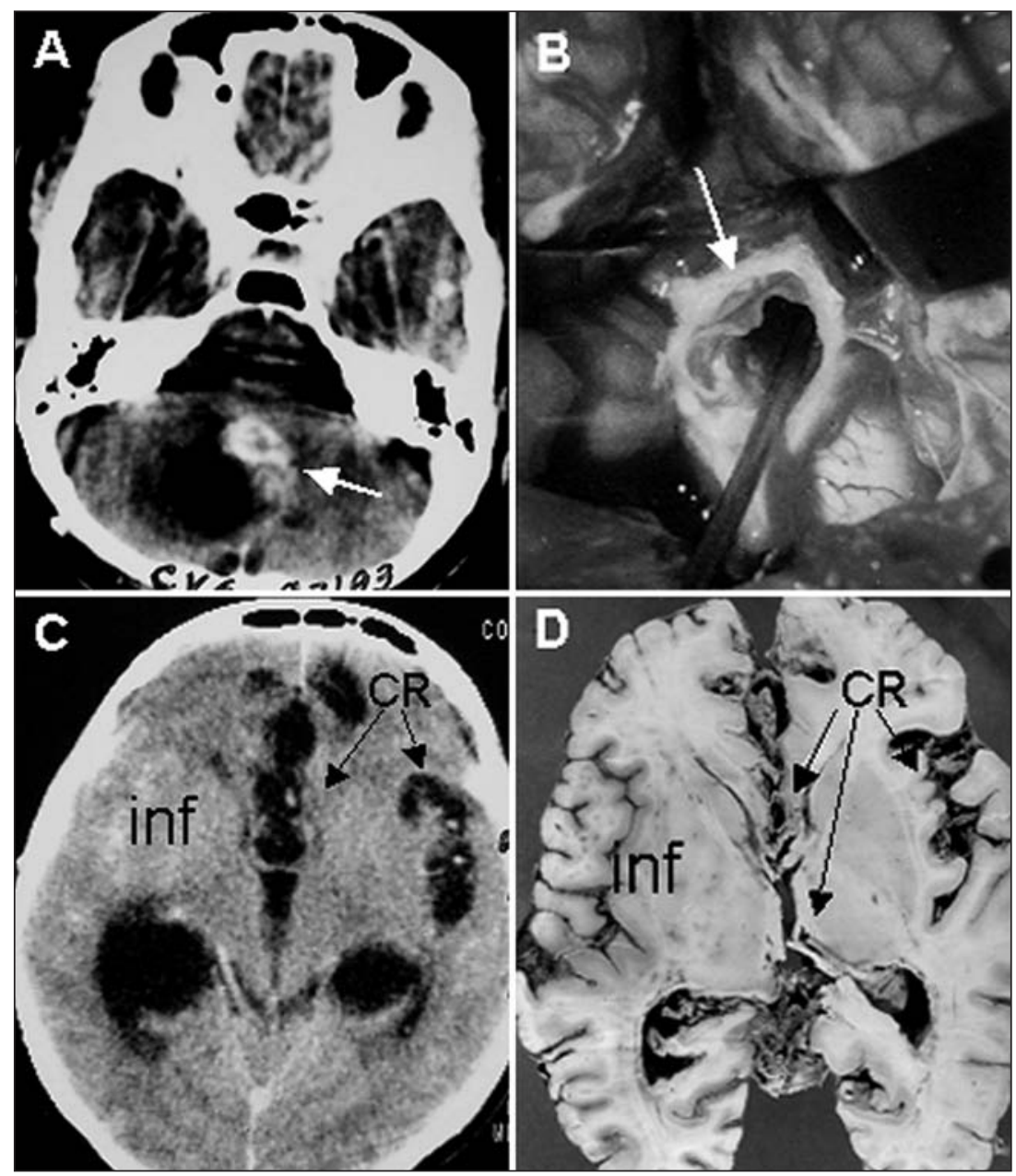

Fig 3. ( $A$ e B) Mulher, 52 a, meningoencefalite + hipertensão intracraniana + síndrome vestibular, síndrome $L C R$ na NCC, DVP com quatro revisões, óbito; $A$ : TC crânio pré-cirurgia de fossa posterior mostra isolamento e deformação cística de $I V^{\circ}$ ventrículo com retenção mural de contraste (seta); (B) Campo cirúrgico após a retirada das traves fibróticas, permanecendo o espessamento da parede ventricular (seta). (C e D) Mulher, 30 a, meningoencefalite + hipertensão intracraniana + cefaléia + epilepsia + depressão, LCR com pleocitose + hiperproteinorraquia + positividade das reações para cisticercose (fixação de complemento e imunofluorescência), DVP sem revisões, óbito; (C) TC crânio com contraste mostra intenso edema cerebral + infarto isquêmico (inf) + cistos racemosos (setas) comprovados em necropsia (D).

bridamento de aracnoidite medular, um para drenagem de hematoma subdural, um para retirada de cisto occipital degenerado com espessamento de cápsula e cinco $(62,5 \%)$ para abordagem direta do IVo ventrículo. Nestes cinco pacientes, além de cistos degenerados retirados em dois deles, verificou-se intenso processo fibrótico (Figs 3A e 3B) em todos. A evolução destes cinco pacientes foi fatal em quatro $(80 \%)$ e seqüelar em um $(20 \%)$.

Apenas cinco $(40,0 \%)$ pacientes que faleceram foram submetidos ao estudo necroscópico em que se evidenciou intensa fibrose cística de IVo ventrículo em três deles; infartos cerebrais extensos (Figs 3C e 3D) por vasculite cisticercótica em dois; presença de cistos cisticercóticos racemosos em degeneração em um e degenerados em outro. A causa básica do óbito foi a HIC em quatro e embolia pulmonar maciça em um.

\section{DISCUSSÃO}

Embora a NCC possa ser assintomática em mais da metade de seus portadores, a HDC, conhecido marcador de mau prognóstico ${ }^{10}$, é uma presença comum nas manifestações graves ${ }^{5,11,12}$. Comporta-se de forma oposta às calcificações do ponto de vista prognóstico, mas constitui, como essas, estágios evolutivos finais da NCC, constituindo expressões da variabilidade na resposta imunológica. Quando essa resposta é mediada no espaço subaracnóideo, há persistência de 
exsudato inflamatório que induz à formação de meningoencefalite que, com o passar do tempo, leva à formação de fibrose e provoca a obstrução da circulação do LCR, desencadeando a formação de hidrocefalia obstrutiva ${ }^{13}$. Essa foi a fisiopatogenia da HDC observada em $81 \%$ dos pacientes estudados.

Na NCC, a reação à distância (Figs 2A, 2B, 3A e 3B) é definida como uma resposta hiperérgica à presença do antígeno, apresentando relação direta com o número e idade dos cisticercos e relação inversa com a capacidade de resistência do organismo invadido ${ }^{3}$. Nos pacientes estudados, a HDC constituiu uma reação à distância do tecido atingido em 42 (89,4\%) deles. Neste grupo, estão os $13(23,4 \%)$ que apresentaram edema cerebral e calcificações associadas à HDC. Ou seja, nenhum desses pacientes apresentou cistos intraventriculares que justificassem uma reação local como resposta à presença de parasita, mostrando que não há necessidade da presença de cistos intraventriculares para o desenvolvimento da HDC, basta que exista antígeno circulante ${ }^{14}$.

Na casuística apresentada, a presença de formas graves e uma evolução pior foi mais freqüente nos homens $(70 \%)$ que nas mulheres, contrariando o que se diz na literatura ${ }^{15,16}$, mas obedecendo a proporção de maior envolvimento do sexo masculino na população brasileira ${ }^{2}$. Essa discrepância baseia-se no fato das manifestações clínicas diferirem de uma região para outra envolvendo fatores sócio-culturais, genéticos, ambientais e nutricionais ${ }^{17-19}$.

No que diz respeito às manifestações clínicas, embora as crises epilépticas sejam mais freqüentes no sexo masculino e a cefaléia, no feminino, esse quadro inverteu-se nos pacientes estudados. A associação de epilepsia e HIC ${ }^{2}$, assim como na maioria dos pacientes com NCC, também foi observada no presente estudo. A presença de crises parciais simples e complexas, com ou sem generalização secundária, foi superior $(91,3 \%)$ à observada na população geral com NCC $(75 \%)^{2}$. Os distúrbios psíquicos como depressão, demência ou manifestações vagas e mal definidas, freqüentemente, associados a alterações sensoriais e de percepção ${ }^{2}$, refletem a situação que existe na HIC, conseqüência mais comum e imediata da reação inflamatória no espaço subaracnóideo ${ }^{16,20}$ onde a HDC exerce um efeito compressivo sobre as estruturas corticais, predominantemente, frontais e temporais, constituindo um sinal de alerta freqüentemente ignorado. As manifestações psiquiátricas em pacientes com HDC podem ser a primeira queixa que leva o paciente ao médico, como ocorreu em dois pacientes nossos (4,2\%) e que, a demora na intervenção levou à evolução fatal. Os distúrbios vasculares ocorreram como infartos cerebrais isquêmicos de proporções variáveis e conseqüentes à vasculite cisticercótica em $17 \%$ dos pacientes analisados, reforçando a necessidade de pesquisar a presença de NCC em pacientes com acidentes vasculares procedentes de áreas endêmicas. O comprometimento medular acometeu, preferencialmente, as mulheres e, na maioria das vezes, às custas de aracnoidite medular, ratificando o fato de que, na NCC, muitas manifestações ocorrem pelas reações à distância conseqüentes ao antígeno circulante $^{3}$. No único caso com cistos medulares, a localização foi extramedular torácica, apresentação de maior freqüência².

A síndrome liquórica da NCC mostrou uma ocorrência maior $(65,9 \%)$ que a média verificada na literatura $(54 \%)^{2}$. Não se observou normalidade no LCR dos pacientes avaliados. A glicorraquia zero, verificada em $17 \%$ de nossos pacientes, não é comum, mas ocorre $^{11} \mathrm{e}$, nesses casos, torna-se mais um importante marcador de mau prognóstico.

Os exames por TC, realizados em 43 pacientes para o diagnóstico e, em cinco, após o diagnóstico de $\mathrm{HDC}$, revelaram que $27,6 \%$ dos pacientes apresentaram, exclusivamente, a forma inativa (contra $79-93 \%$ da literatura) $)^{2}$ e, 44,7\%, exclusivamente, a forma ativa (contra $5-40 \%$ da literatura) ${ }^{2}$, sugerindo que a HDC por NCC possa ser tanto uma resultante de um processo inflamatório reacional subagudo à presença de cisticercos viáveis, como um processo seqüelar fibrótico. Tudo depende da interação parasita-hospedeiro e da resposta imunológica individual de cada paciente.

As DVP constituem tratamento paliativo, e podem evoluir com exacerbação da NCC e elevada mortalidade, mesmo quando os cistos não são detectados à $\mathrm{TC}^{21}$. O prognóstico é pobre e apresenta elevados índices de complicações manifestas por obstruções e infecções do sistema de DVP21,22, como ocorreu em $16 \%$ dos pacientes estudados. Constatamos uma necessidade maior de revisões de DVP (54\%) com número maior de revisões por paciente ( 1 a 7) em relação à literatura (29\% e 1 a 3, respectivamente) ${ }^{22}$. Verificamos que, embora não houvesse indicação de interposição de DVP antes da terapêutica cisticida, aqueles pacientes que foram submetidos a esse tipo de cirurgia, durante ele, já mostravam sinais discretos, à TC ou RM, de edema transependimário sugestivo de HIC incipiente. Talvez suas evoluções tivessem sido melhores se tal observação fosse valorizada e, 
conseqüentemente, as DVPs instaladas antes da terapêutica cisticida, considerando que a interposição de DVP seguida de um esquema cisticida favorece evolução melhor da DVP com freqüência 3 vezes menor de complicações ${ }^{23}$. A evolução fatal ocorreu, em média, 12 meses após DVP e/ou terapêutica cisticida, tempo inferior ao relatado na literatura ${ }^{2,4,5,15,16}$. O fator terminal da morte seja através de necropsia, seja pelo quadro clínico, foi a HIC resultante do edema cerebral e/ou hidrocefalia hipertensiva.

A reversibilidade dos sinais e sintomas observados em pacientes com evolução satisfatória e até mesmo em alguns que tiveram seqüelas, pode indicar que não existiu uma lesão destrutiva ${ }^{24}$, mas sim um distúrbio metabólico decorrente do edema periventricular ou estase do fluxo normal no espaço extracelular, diretamente contra o sistema ventricular ${ }^{25}$.

$\mathrm{O}$ estudo retrospectivo de 47 pacientes do HCUNESP de Botucatu - SP, selecionados e com diagnóstico de HDC por NCC, permite concluir que: 1. Houve maior incidência desta manifestação neurológica em homens na faixa etária mais produtiva de suas vidas; 2 . A meningoencefalite e os distúrbios psíquicos constituíram associação freqüente; 3 . Os distúrbios psiquiátricos, principalmente a depressão, deveriam ter sido considerados sinais de alerta para o diagnóstico de HDC, por tratar-se de pacientes procedentes de área endêmica; 4. Meningoencefalites de repetição foram manifestações importantes $(80,9 \%)$ na gênese das HDC; 5 . O comprometimento medular esteve associado à HDC naqueles pacientes com diminuição de força em membros inferiores, espasticidade e hiperreflexia; 6 . A maioria $(65,9 \%)$ apresentou LCR com a síndrome completa da NCC; 7. A presença exclusiva de cistos viáveis, não incluindo as formas racemosas, ou de calcificações ocorreu com uma freqüência semelhante (respectivamente, 23,4 e 27,6\%); 8. Para a conclusão diagnóstica, a RM foi necessária em $52,4 \%$ dos pacientes; 9 . A interposição da DVP antes do esquema cisticida contribuiu para uma evolução melhor; 10 . O mau prognóstico esteve diretamente relacionado ao número de revisões de DVP e à formação de IVo ventrículo isolado.

Agradecimentos - Os autores agradecem, ao Prof. Titular Paulo Roberto Curi, a inestimável colaboração na orientação e avaliação estatística dos resultados deste estudo.

\section{REFERÊNCIAS}

1. Prockop L. Hidrocefalia. In Rowland LP. Merrit - Tratado de Neurologia. 10.Ed. Tradução de Araújo CLC. Rio de Janeiro: Editora Guanabara-Koogan, 2000: 238-244.

2. Agapejev S. Aspectos clínico-epidemiológicos da neurocisticercose no Brasil: análise crítica. Arq Neuropsiquiatr 2003;61:822-828.

3. Trétiakoff C, Silva ACP. Contribuição para o estudo da cysticercose cerebral em particular das lesões tóxicas à distancia n'esta affecção. Mem Hosp Juquery 1924;1:37-66.

4. Colli BO, Martelli N, Assirati JA Jr, et al. Cysticercosis of the central nervous system. I. Surgical treatment of cerebral cysticercosis: a 23 years experience in the Hospital das Clínicas of Ribeirão Preto Medical School. Arq Neuropsiquiatr 1994;52:166-186.

5. Agapejev S, Silva MD, Ueda AK. Severe forms of neurocysticercosis: treatment with albendazole. Arq Neuropsiquiatr 1996;54:82-93.

6. Del Brutto $\mathrm{OH}$, Wadia NH, Dumas M, et al. Proposal of diagnosis criteria for human cysticercosis. J Neurol Sci 1996;142:1-6.

7. Evans WA. An encephalographic ratio for estimating ventricular enlargement and cerebral atrophy. Arch Neurol Psychiatry 1942;47:931-937.

8. Spina-França A. Síndrome liquórica da neurocisticercose. Arq Neuropsiquiatr 1961;19:307-314.

9. Commission on Classification and Terminology of the International League Against Epilepsy. Proposal for revised classification of epilepsies and epileptic syndromes. Epilepsia 1989;30:389-399.

10. Estañol B, Kleriga E, Loyo M, et al. Mechanisms of hydrocephalus in cerebral cysticercosis: implications for therapy. Neurosurgery 1983; 13:119-123.

11. Rogel-Ortiz F, Vera-Pedro M. Meningitis cisticercosa. Gac Méd Méx 1997;133:301-305.

12. Estañol B, Corona T, Abad P. A prognostic classification of cerebral cysticercosis: therapeutic implications. J Neurol Neurosurg Psychiatry 1986; 49:1131-1134

13. Mise B, Klarica M, Seiwerth S, Bulat M. Experimental hydrocephalus and hydromyelia: a new insight in mechanism of their development. Acta Neurochir (Wien) 1996;138:862-869.

14. Garcia HH, Gonzalez AE, Gilman RH, et al. Cysticercosis Working Group in Peru. Circulating parasite antigen in patients with hydrocephalus secondary to neurocysticercosis. Am J Trop Med Hyg 2002; 66:427-430.

15. Lobato RD, Lamas E, Portillo JM, et al. Hydrocephalus in cerebral cysticercosis: pathogenic and therapeutic considerations. J Neurosurg 1981; 55:786-793.

16. Sotelo J, Marin C. Hydrocephalus secondary to cysticercotic arachnoiditis. J Neurosurg 1987;66:686-689.

17. Fleury A, Beltrán C, Ferre E, et al. Application of synthetic peptides to the diagnosis of neurocysticercosis. Trop Med Internat Health 2003;8: 1-7.

18. Biondi GF, Padilla A, Beltran CJ, et al. Cisticercosis: identificación de epitopes inmunogenicos del antigeno TCA 5.5. (Resumo). Anais XIII Congresso Latino-Americano de Parasitologia 1997:21.

19. Thussu A, Sehgal S, Sharma M, et al. Comparison of cellular response in single- and multiple-lesion neurocysticercosis. Ann Trop Med Parasitol 1997;91:627-632.

20. Salazar A, Sotelo J, Martinez H, Escobedo F. Differential diagnosis between ventriculitis and fourth ventricle cyst in cysticercosis. J Neurosurg 1983;59:660-663.

21. Cuetter AC, Garcia-Bobadilla J, Guerra LG, Martinez FM, Kaim B. Neurocysticercosis: focus on intraventricular disease. Clin Infect Dis 1997; 24:157-164.

22. Choksey MS, Malik IA. Zero tolerance to shunt infections: can it be achieved? J Neurol Neurosurg Psychiatry 2004:75:87-91.

23. KelleY R, Duong DH, Locke GE. Characteristics of ventricular shunt malfunctions among patients with neurocysticercosis. Neurosurgery 2002;50:757-762.

24. Lönnum A, Kornstad S. Long-term prognosis of patients with central cerebral ventricular enlargement: a fourth follow-up of 100 patients with a $3^{\text {rd }}$ ventricle measuring $12 \mathrm{~mm}$ or more in width. Acta Neurol Scandinav 1974;50:409-433.

25. Wikkelsö C, Anderson H, Blomstrand C, Matousek M, Svendsen P. Computed tomography of the brain in the diagnosis and prognosis in normal pressure hydrocephalus. Neuroradiology 1989;31:160-165. 